\title{
Desarrollo y evaluación de la competencia comunicativa en la formación inicial de maestros
}

\author{
Marta Gràcia, Maria-Josep Jarque, Marta Astals y Kholoud Rouaz
}

\begin{abstract}
RESUMEN
La educación de los futuros maestros necesariamente debe incluir el desarrollo de la competencia comunicativa por su carácter fundamental como instrumento de comunicación, de producción y transformación del conocimiento y para la mejora de la calidad de los aprendizajes, así como para favorecer el desarrollo del pensamiento crítico, tanto en relación con los propios estudios como con su posterior desempeño profesional. A pesar de ello, son pocos los estudios e investigaciones dedicados a ello. El propósito de este artículo es contribuir a llenar este vacío mediante la presentación de los resultados de un estudio, cuyo objetivo estriba en la mejora de la construcción de textos orales argumentativos y la reflexión metalingüística sobre sus elementos, mediante el desarrollo e introducción de estrategias e instrumentos docentes. Se utilizó un método mixto, que incluye análisis cuantitativos y cualitativos. Los instrumentos utilizados fueron la EVALOE o Escala de Evaluación de la Lengua Oral en contexto Escolar (Gràcia et al., 2015a) y una rúbrica construida al efecto. Los resultados muestran que si se introducen estrategias e instrumentos docentes ajustados, los estudiantes aprenden a construir textos (monogestionados y plurigestionados) y a reflexionar sobre ellos.
\end{abstract}

Palabras clave: competencia comunicativa, aprendizaje, evaluación, formación docente, España.

\section{Marta Gràcia}

mgraciag@ub.edu

Española. Doctora en Psicología por la Universidad de Barcelona, España. Profesora titular en el Departamento de Cognición, Desarrollo y Psicología de la Educación, Universidad de Barcelona. Temas de investigación: enseñanza y aprendizaje de la lengua oral en contexto escolar y universitario, instrumentos de evaluación y de formación docente en lengua oral.

\section{Maria-Josep Jarque}

mj_jarque@ub.edu

Española. Máster en Lingüística, Licenciada en Filología. Profesora asociada en el Departamento de Cognición, Desarrollo y Psicología de la Educación de la Universidad de Barcelona, España. Temas de investigación: enseñanza y aprendizaje de lenguas en la modalidad oral y signada y en la estructura lingüística de las lenguas de signos.

\section{Marta Astals}

martastals@gmail.com

Española. Estudiante del grado de Psicología en la Universidad de Barcelona, España. Investigadora en formación en el Departamento de Cognición, Desarrollo y Psicología de la Educación de la Universidad de Barcelona. Temas de investigación: enseñanza y aprendizaje de la lengua oral en contexto universitario.

\section{Kholoud Rouaz}

krouaz.imp@gmail.com Marroquí. Estudiante del grado de Psicología en la Universidad de Barcelona, España. Investigadora en formación en el Departamento de Cognición, Desarrollo y Psicología de la Educación de la Universidad de Barcelona. Temas de investigación: enseñanza y aprendizaje de la lengua oral en contexto universitario. 


\title{
Desenvolvimento e avaliação da competência comunicativa na formação inicial de professores
}

\section{RESUMO}

A educação dos futuros professores necessariamente deve incluir o desenvolvimento da competência comunicativa por seu carácter fundamental como instrumento de comunicação, de produção e transformação do conhecimento e para a melhora da qualidade das aprendizagens, assim como para favorecer o desenvolvimento do pensamento crítico, tanto em relação com os próprios estudos como com seu posterior desempenho professional. A pesar disso, são poucos os estudos e investigações dedicadas a isso. O propósito deste artigo é contribuir com o preenchimento deste vazio mediante a apresentação dos resultados de um estudo cujo objetivo se sustenta na melhora da construção de textos orais argumentativos e na reflexão metalinguística sobre seus elementos, mediante o desenvolvimento e introdução de estratégias e instrumentos docentes. Se utilizou um método misto, que inclui análises quantitativas e qualitativas. Os instrumentos utilizados foram a EVALOE ou Escala de Avaliação da Língua Oral em contexto Escolar (Gràcia et al., 2015a) e uma rubrica construída para efeito. Os resultados mostram que se se introduzem estratégias e instrumentos docentes ajustados, os estudantes aprendem a construir textos (solilóquio e dialogo) e a refletir sobre eles.

Palavras chave: competência comunicativa, aprendizagem, avaliação, formação docente, Espanha.

\section{Development and assessment of communicative competence in initial teacher training}

\begin{abstract}
The education of future teachers must necessarily include the development of communicative competence because of its fundamental character as an instrument of communication, of the production and transformation of knowledge and for the improvement of the quality of learning, as well as to favor the development of critical thinking, both in relation to one's own studies and to one's subsequent professional performance. In spite of this, there are few studies and researches dedicated to it. The purpose of this article is to contribute to filling this void by presenting the results of a study whose objective is to improve the construction of argumentative oral texts and metalinguistic reflection on their elements, through the development and introduction of teaching strategies and instruments. A mixed method was used, which includes quantitative and qualitative analyses. The instruments used were the EVALOE or Escala de Evaluación de la Lengua Oral en Contexto Escolar (i. e. Oral Language Assessment Scale in a School Context) (Gràcia et al., 2015a) and a rubric constructed for this purpose. The results show that if adjusted teaching strategies and instruments are introduced, students learn to construct texts in which one or more interlocutors are involved and to reflect on them.
\end{abstract}

Key words: communicative competence, learning, assessment, teacher training, Spain. 


\section{Introducción}

La competencia comunicativa (hablar y escuchar) forma parte actualmente de la mayoría de currículos, desde la educación infantil hasta la universidad. Sin embargo, en todos los niveles educativos, exceptuando la educación infantil, los objetivos y contenidos vinculados al desarrollo de la competencia comunicativa en todas sus dimensiones (gestión de la conversación, argumentación, cohesión, coherencia, etcétera) se trabajan, habitualmente, de manera poco sistemática y explícita, excepto en las presentaciones orales formales. Los alumnos son, en general, poco conscientes de que durante la mayor parte de la jornada escolar o de las clases universitarias la lengua oral juega, o podría jugar, un papel crucial en el aprendizaje de los diferentes contenidos y en el desarrollo de otras capacidades, como la de aprender a aprender.

\section{Diseño de entornos comunicativos en el aula}

La competencia comunicativa de los maestros es crucial para la creación de ambientes ricos que ayuden a los alumnos a aprender los contenidos del currículo de una manera constructiva y a desarrollar su lenguaje (Justice y Ezell, 1999; Marinac et al., 2008; Mercer, 2010; Gràcia et al., 2015b). A través de la implementación del currículo, especialmente de aquellos objetivos y contenidos vinculados a la construcción de textos y la pragmática, se espera que los alumnos acaben la etapa de educación primaria (11-12 años) habiendo alcanzado una competencia pragmática en su primera lengua, en la lengua o lenguas vehiculares de los contenidos académicos, en la segunda lengua de la escuela (si procede), así como en la lengua o lenguas extranjeras, que les permita preguntar, pedir explicaciones, hacer demandas de información, cuestionar, plantear dudas, argumentar, rebatir, reflexionar sobre el lenguaje en situaciones diversas, más o menos formales o académicas, en relación con contenidos (relacionados con las matemáticas, lengua, experiencias personales, mundo en el que viven, música, aficiones, etcétera), interlocutores, contextos y objetivos diversos (Gràcia et al., 2017; Generalitat de Catalunya, 2013; Ishihara y Cohen, 2014).

Todas estas funciones y habilidades comunicativas deberían poderlas poner en juego en el marco de textos orales no sólo adecuados al contexto, sino ricos y complejos desde el punto de vista semánticopragmático y formal (fonética-fonología, morfología, sintaxis y estructura textual). Esta meta únicamente puede ser alcanzada si en la escuela (así como también en otros contextos) se facilita a los alumnos utilizar el lenguaje para interactuar de manera dialógica (Mercer, 2010; Kathard et al., 2015). La interacción tendría lugar en situaciones en las que se sientan seguros, en las que puedan solicitar ayuda, en las que los compañeros, el docente y otros profesionales puedan ser modelos a imitar, en las que se utilicen estrategias que contribuyan al desarrollo de estas habilidades y de la competencia comunicativa, y en las que se promueva la reflexión sobre el lenguaje y su uso, es decir, la competencia metalingüística (Hoffman, 2011; Swain y Lapkin, 2013).

\section{La competencia comunicativa en la formación inicial de maestros}

Para conseguir que los alumnos en las etapas de educación infantil y primaria desarrollen las habilidades a las que se acaba de hacer referencia en el apartado anterior, es necesario que sus maestros sean conscientes de esta necesidad y que, al mismo tiempo, hayan desarrollado competencias para ayudar a sus alumnos a desarrollarlas.

Tal como señala Peña (2008) en un trabajo sobre competencias básicas en educación superior, y específicamente sobre la competencia oral y escrita, el valor de la lectura, la escritura y la expresión oral no radica solamente en su condición de medios para acumular información o para demostrar el conocimiento adquirido en la universidad, sino, fundamentalmente, como instrumentos poderosos para producir y transformar el conocimiento, mejorar 
la calidad de los aprendizajes, desarrollar el pensamiento crítico de los estudiantes y hacerlos partícipes en su proceso de formación. Además de su contribución a los citados logros académicos, la capacidad para comunicar las ideas de una manera clara y convincente en forma oral, en el caso de los estudiantes que se preparan para trabajar de maestros, es una condición indispensable para su desempeño profesional, puesto que van a tener que ayudar a sus propios alumnos a desarrollar esta capacidad.

En un estudio realizado sobre el desarrollo de las competencias orales y escritas en el marco del Espacio Europeo de Educación Superior (EEES) (RuízMuñoz, 2012), se pone de relieve la carencia de publicaciones en las que se describan experiencias centradas en la competencia oral y escrita de los estudiantes universitarios en el marco del EEES. Coincidimos con la autora en que conviene apostar por la puesta en marcha de iniciativas de investigación e innovación acerca del uso de estrategias vinculadas a la construcción de textos orales en el aula, orientadas al desarrollo profesional.

Monarca (2013), en un trabajo de reflexión sobre la importancia de fomentar la participación de los estudiantes universitarios en las clases como elemento de construcción del pensamiento crítico, señala que este enfoque conlleva una serie de concepciones sobre la construcción del discurso didáctico (Acosta, 2012), la construcción del conocimiento como producto social compartido, la construcción del conocimiento como proceso subjetivo y de aprendizaje del sujeto, por tanto, una idea sobre cómo se aprende y cómo se enseña. Coincidimos con el autor en que esta participación, entendida en su mayor parte como participación a partir de intervenciones orales en las clases, supone entender que se trata de una práctica y una competencia que se aprende, cuyo aprendizaje forma parte de la historia del sujeto y que, por tanto, éste debe ser contemplado por quien enseña, lo cual requiere que forme parte de la propuesta didáctica, de la acción educativa.
En los últimos 20 años esta competencia ha sido uno de los contenidos más importantes de la formación inicial de maestros (FIM) (Ogienko y Rolyak, 2009). En un estudio de revisión llevado a cabo por Zlatić y colaboradores (2014) en el que se compara la competencia comunicativa entre cuatro tipos de maestros diferentes y un grupo de personas con otra formación, se pone de relieve que existen pocas diferencias respecto a la competencia comunicativa entre maestros y no maestros, tampoco entre estudiantes de maestro que han participado en el entrenamiento de habilidades comunicativas y aquellos que no lo han hecho, con algunas excepciones. Los autores concluyen que es necesario diseñar programas específicos para mejorar la competencia comunicativa de maestros en activo y otras para maestros en formación.

$\mathrm{El}$ análisis de los datos recogidos en una primera fase del estudio que se presenta a partir de entrevistas a maestros en activo y en formación, así como a docentes universitarios, de observaciones en aulas de infantil y primaria, y de la revisión de planes docentes del grado de Maestro, sugiere la necesidad de introducir cambios en la FIM con el fin de ayudar a los estudiantes a mejorar su competencia comunicativa, como estudiantes universitarios y como futuros maestros (Gràcia et al., 2015c, 2015d, 2016, 2017). Los estudiantes necesitan tomar conciencia (y lo han hecho durante las entrevistas) de que la habilidad de intervenir en clase, durante la revisión de conocimientos previos, durante el trabajo en pequeño grupo en clase ( $\mathrm{Li}$ et al., 2015), en las puestas en común posteriores, es fundamental para dar sentido a los contenidos y para aprenderlos con un elevado grado de significatividad (Coll, 2013), y ello incluye la reflexión sobre sus competencias para gestionar una conversación, para generar textos coherentes y cohesionados, para argumentar sus opiniones, para contraargumentar o refutar las de los compañeros o el profesor, para formular preguntas que enriquezcan la discusión, etcétera. 
Como futuros maestros, estas estrategias les serán útiles para convertir las clases en entornos comunicativos en los que los alumnos participen, pregunten, gestionen, reflexionen sobre el lenguaje para mejorar su competencia comunicativa y para dar sentido al aprendizaje de todos los contenidos. También les será útil para participar, posteriormente, y en el practicum durante la etapa de formación, de forma activa en las reuniones de ciclo, de claustro, con los especialistas (psicopedagogos, orientadores, logopedas, etcétera). Las reuniones con las familias, en toda su diversidad, sin duda también se verán enriquecidas, si sus habilidades conversacionales y de gestión son buenas.

A partir de los resultados que acabamos de revisar, que han puesto de manifiesto la necesidad de introducir cambios en la manera como se trabaja la lengua oral en la FIM, nos proponemos probar la eficacia de algunos instrumentos, recursos y estrategias con el fin de mejorar la competencia comunicativa y lingüística de los estudiantes universitarios de los grados de maestro de educación infantil y de educación primaria. Para ello, hemos llevado a cabo un estudio piloto que se plantea los objetivos siguientes. En primer lugar, nos proponemos analizar la contribución de una intervención en contexto universitario a la mejora de las habilidades de conversación de los estudiantes, especialmente argumentativas, cuando trabajan en grupos cooperativos en el aula. En segundo lugar, nos proponemos explorar el grado en el que esta intervención favorece el desarrollo de habilidades discursivas y argumentativas durante las discusiones en gran grupo, antes o después de realizar alguna actividad en grupo cooperativo. Finalmente, nos proponemos analizar la manera como la intervención contribuye a mejorar las habilidades de los estudiantes para construir textos orales monogestionados que forman parte del proceso de evaluación de la asignatura.

Concretamente, las preguntas de investigación que nos formulamos son las siguientes:
- ¿Se van a percibir diferencias entre los textos orales que se construyan en las sesiones de clase de los docentes que promuevan la participación oral de los estudiantes de manera sistemática y planificada y aquellos que no lo hagan?

- ¿Se van a detectar diferencias entre los textos orales que se construyan en las sesiones de clase en función del momento dentro de la unidad temática en el que se produzcan, es decir, entre las clases de inicio de bloque y final en un mismo grupo clase?

- ¿Las conversaciones que se generen en los grupos pequeños de los grupos clase en los que los docentes promueven la participación oral de los estudiantes de manera sistemática y planificada van a ser diferentes de aquellos grupos pequeños en los que los docentes no lo hacen?

\section{Metodología}

Se trata de un estudio de caso con claros componentes de investigación-acción, dado el rol íntegramente participativo de dos de las docentes (Latorre, 2003; Riba, 2009). Los principales agentes implicados en el estudio son docentes e investigadoras al mismo tiempo, que persiguen mejorar su práctica educativa, así como la comprensión de la misma en aras de contribuir a la mejora de la competencia comunicativa y lingüística de sus estudiantes. La estrategia utilizada puede considerarse dentro de un enfoque de métodos mixtos en tanto que el estudio recoge y analiza datos cualitativos y cuantitativos (Burke et al., 2007).

\section{Participantes}

Han participado en esta investigación cuatro docentes universitarios (dos del grado de Educación Infantil (EI) y dos del grado de Educación Primaria (EP)) y sus grupos de estudiantes (entre 48 y 54 por grupo) de la asignatura anual Psicología de la Educación, de primer curso, con dos sesiones de clase de dos horas semanales. Una docente de cada grado ha introducido innovaciones en sus clases (dos grupos de innovación 
o GI) y los otros dos han realizado sus clases de la manera habitual (dos grupos de comparación o GC). Una de las docentes ha coordinado la investigación trabajando conjuntamente con la otra docente que ha introducido innovaciones, y una tercera investigadora predoctoral. Conjuntamente, las tres han participado en el diseño, el seguimiento y reajustes de la innovación. Las investigadoras predoctorales y una estudiante de grado han proporcionado apoyo en los registros de video de clases y en el almacenado del material registrado y recogido. En el análisis de datos han participado otras tres estudiantes de grado. Las observaciones se han realizado en las aulas de la Facultad de Educación de la Universidad de Barcelona.

\section{Instrumentos}

Para la recogida de datos se han elaborado diferentes instrumentos ad hoc y se han utilizado otros ya publicados y validados.

\section{La discusión argumentativa en el aula como recurso para enseñar y aprender: la Metodología Conversacional}

Desde la Metodología Conversacional (MG) (Gràcia et al., 2017) se enfatiza la necesidad de que los objetivos y contenidos vinculados a la capacidad para escuchar, hablar y conversar estén presentes en el programa de todas las materias, donde deben definirse los objetivos funcionales a conseguir, así como los sistemas de enseñanza, seguimiento y evaluación del aprendizaje que van a ser utilizados. En este estudio, la MC se concreta en las siguientes estrategias:

- Trabajar los contenidos de los diferentes bloques temáticos otorgando especial importancia a la lengua oral, tanto desde el punto de vista expresivo (hablar) como comprensivo (escuchar). Por ejemplo, las docentes de los grupos de innovación plantean las clases como una discusión sobre los textos, de manera que sea necesario que los estudiantes participen activamente para avanzar en la comprensión de los contenidos de los bloques temáticos.

- Promover la competencia metalingüística de los estudiantes, mediante la reflexión sobre los usos y funciones de las construcciones lingüísticas en la interacción y su adecuación al contexto. En este caso las docentes de los grupos de innovación animan a los estudiantes a reflexionar sobre la manera como utilizan la lengua oral durante las clases y en su adecuación, a través de la propuesta del uso de instrumentos como las rúbricas.

- Otorgar relevancia al contexto comunicativo y su gestión; explicitar los objetivos que se pretenden alcanzar; y utilizar estrategias para reformular las producciones de los estudiantes y para promover el uso del lenguaje con diferentes intenciones (argumentar, preguntar, aclarar, sugerir, contrastar, etcétera).

- Incorporar elementos vinculados a la construcción de textos orales con especial referencia a la conversación argumentativa: exposición de argumentos, contraargumentos, refutaciones o replicas; composición de textos que incorporen recursos que contribuyan a la coherencia y cohesión textual; uso de léxico general preciso y de la terminología específica del área, etcétera. Esto supone que en los grupos de innovación las docentes introducen actividades en las clases que promueven la discusión entre los estudiantes, como preguntas que supongan posicionarse ante alguna cuestión relacionada con la temática y utilizar para ello el léxico que se está trabajando.

- Trabajar en grupos pequeños cooperativos.

- Incorporar a la evaluación de la asignatura la construcción de textos argumentativos monogestionados y plurigestionados registrados en video. Por ejemplo, en los grupos de innovación docente, un porcentaje de la nota de cada bloque se vincula con la construcción de un texto oral (registro en video) sobre el que se 
discute previamente en el aula, de manera que la docente orienta a los estudiantes para que se ajuste a unas determinadas características. Las docentes proponen a los estudiantes la simulación de situaciones reales que se pueden encontrar en un futuro como maestros.

\section{Instrumentos específicos para evaluar la discusión argumentativa como recurso para enseñar $y$ aprender}

Instrumento de seguimiento, registro y evaluación de la conversación argumentativa. Este instrumento, construido ad hoc para esta investigación, tiene como objetivo observar, registrar y evaluar la competencia comunicativa y lingüística relacionada con la conversación argumentativa en pequeño grupo y en grupo clase. Incluye dos elementos: 1) una rúbrica, que comprende dimensiones, indicadores, una breve explicación de indicadores y una breve explicación de cada uno de los descriptores (del 1 al 4); 2) un documento de consulta de una extensión de 15 páginas, en el que se recogen los fundamentos teóricos que están en la base de la rúbrica, así como ejemplos de recursos lingüísticos y de textos orales y escritos que los incluyen. En la tabla 1 se presentan las dimensiones e indicadores.

Tabla 1. Dimensiones e indicadores de la rúbrica

\begin{tabular}{|c|c|c|c|c|c|}
\hline Dimensiones & Indicadores & 1 & 2 & 3 & 4 \\
\hline \multirow{4}{*}{$\begin{array}{l}\text { Gestión de la } \\
\text { interacción }\end{array}$} & Marcadores interactivos & & & & \\
\hline & Turnos comunicativos & & & & \\
\hline & Gestión de la participación & & & & \\
\hline & Estrategias de cortesía & & & & \\
\hline \multirow{3}{*}{ Multimodalidad } & Gestos manuales & & & & \\
\hline & Gestos batutas & & & & \\
\hline & Gestos faciales y corporales & & & & \\
\hline \multirow{2}{*}{ Prosodia } & Entonación & & & & \\
\hline & Locución & & & & \\
\hline \multirow{3}{*}{ Coherencia } & Organización de la información & & & & \\
\hline & Exposición, agrupamiento y secuencia de las ideas & & & & \\
\hline & Relevancia, sentido unitario, carácter completo de la información & & & & \\
\hline \multirow{3}{*}{ Cohesión textual } & Conexión entre fragmentos & & & & \\
\hline & Conexión entre oraciones & & & & \\
\hline & Tratamiento de la información & & & & \\
\hline \multirow{7}{*}{$\begin{array}{l}\text { Estrategias } \\
\text { argumentativas }\end{array}$} & Tesis & & & & \\
\hline & Validez de la argumentación & & & & \\
\hline & Contraargumentos & & & & \\
\hline & Falacias & & & & \\
\hline & Conclusión & & & & \\
\hline & Construcciones evidenciales & & & & \\
\hline & Patrones de interacción de la secuencia argumentativa & & & & \\
\hline \multirow{2}{*}{$\begin{array}{l}\text { Léxico y } \\
\text { terminología }\end{array}$} & Léxico común & & & & \\
\hline & Terminología del área de conocimiento & & & & \\
\hline
\end{tabular}


Como se aprecia en la tabla 1, la rúbrica está organizada en siete dimensiones. La dimensión Gestión de la interacción hace referencia a la capacidad para gestionar la interacción en red. Comprende cuatro subdimensiones: 1) el uso de marcadores interactivos que favorecen la participación en red, tanto lingüísticos como gestuales (Mondada, 2013; Selting, 2010; Schegloff, 2015); 2) la participación en turnos comunicativos (Kuhn et al., 2013), 3) la gestión de la participación, y 4) el uso de estrategias de cortesía (Calsamiglia y Tusón, 1999; Cuenca, 2007).

La segunda dimensión remite a la capacidad de poner en marcha los recursos multimodales que caracterizan la interacción social. Incluye el uso de gestos que contribuyen cualitativamente a la expresión verbal: gestos manuales - emblemas, ilustradores y batutas-, faciales y corporales (Müller et al., 2013, 2014; Payrató, 2014; Payrató y Teßendorf, 2013).

En relación con la tercera dimensión, ésta hace referencia al uso de la prosodia, es decir, a la capacidad de poner la locución al servicio de la expresión clara del contenido y la transmisión de emociones. Comprende dos subdimensiones: 1) el uso de la entonación en las diferentes construcciones lingüísticas - curvas entonativas y acento prosódico-, y 2) las características que presenta la locución, tanto en relación con elementos prosódicos como paralingüísticos - la intensidad, la velocidad, el tono y la articulación (Firth, 1957; Selting, 2010).

En cuanto a la cuarta dimensión, ésta evalúa la $c 0^{-}$ herencia, es decir, la exposición, agrupación y secuencia de las ideas que proporcionan un sentido unitario y un carácter completo al contenido de la información (Bublitz, 2011; Charaudeau y Maingueneau, 2002; Verschueren, 1999). La cohesión textual es el objeto de análisis de la quinta dimensión. Se analiza tanto la conexión entre fragmentos y oraciones mediante conectores, como el tratamiento de la información mediante marcadores discursivos u operadores modales (Cuenca, 1995; Halliday y Hasan, 1976; Lo Cascio, 1998; Gràcia et al., 2015; Renkema, 2004).
La sexta dimensión incluye los elementos que remiten a la capacidad de formular y argumentar razonadamente una posición con el objetivo de llegar a un consenso. Presenta siete subdimensiones: formulación de la tesis, validez de los argumentos, exposición de contraargumentos, identificación de falacias, formulación de conclusiones, uso de construcciones evidenciales que indiquen la fuente de la información y uso de patrones en la secuencia argumentativa (Blair y Johnson, 1987; Cano, 2010; Kuhn, 1991; Felton et al., 2015; Leitão, 2000, 2011; Perelman y Olbrechts-Tyteca, 1989; Rapanta y Walton, 2016; Toulmin, 1958; Van Dijk, 1983; van Eemeren et al., 2002; Walton, 2006, 2013; Weinstock et al., 2004).

La última dimensión remite a la capacidad de expresión precisa y variada teniendo en cuenta dos subdimensiones: el léxico común y la terminología del área (Cabré, 1999; Cruse, 2011).

Escala de valoración de la enseñanza de la lengua oral en contexto escolar (EVALOE). Se trata de una escala formada por 30 ítems agrupados en tres subescalas que pueden ser puntuados del 1 al 3. Es una escala validada en contexto de educación infantil y primaria (Gràcia et al., 2015a, 2015b) que se usará por primera vez en contexto universitario para valorar la manera como los profesores participantes enseñan lengua oral en clase (ver tabla 2). El origen de este instrumento se remonta a la necesidad de explicar de manera objetiva la mejora que se había producido en las actuaciones de dos maestras que participaron en una investigación en la que se introdujo una innovación docente (Gràcia et al., 2012), que consistió en ayudarles a usar estrategias que favoreciesen la participación de los alumnos durante las actividades de clase. Con base en las tres dimensiones en las que se agrupaban las estrategias que se sugerían a las docentes, se elaboraron ítems que se fueron revisando hasta que se consensuaron 30 ítems con tres opciones de respuesta. Posteriormente, este instrumento fue validado con un total de 130 observaciones de docentes de niveles educativos y tipologías de escuelas diversos. 
Tabla 2. Subescalas que forman la EVALOE (Gràcia et al., 2015a)

\begin{tabular}{|l|l|c|}
\hline \multicolumn{1}{|c|}{ Subescalas } & \multicolumn{1}{c|}{ Descripción } & Puntuación \\
\hline $\begin{array}{l}\text { Contexto y gestión de la } \\
\text { comunicación (7 ítems) }\end{array}$ & Evalúa de manera global la organización del contexto aula y de la comunicación (contexto físico y normas) & $-/ 24$ \\
\hline $\begin{array}{l}\text { Diseño instruccional } \\
\text { (8 ítems) }\end{array}$ & $\begin{array}{l}\text { Evalúa aquellos objetivos, actividades y la evaluación en relación con la lengua oral, para ver si se incorpora } \\
\text { dicha información y si se hace explíita en las programaciones }\end{array}$ & $-/ 21$ \\
\hline $\begin{array}{l}\text { Funciones comunicativasy } \\
\text { estrategias (15 ítems) }\end{array}$ & $\begin{array}{l}\text { Evalúa cómo el maestro enseña a utilizar los recursos de la lengua oral para determinados propósitos } \\
\text { sociales (informar, preguntar y otros), así como el uso de ciertas estrategias educativas para promover la } \\
\text { expresión de los alumnos }\end{array}$ & $-/ 45$ \\
\hline TOTAL & & $-/ 90$ \\
\hline
\end{tabular}

\section{Procedimiento de recogida de datos}

El proceso de recogida de datos se ha desarrollado siguiendo una serie de pasos. En primer lugar, se solicitó el permiso a las directoras de estudios del grado de EI y de EP. En segundo lugar, la investigadora principal (IP) se reunió con las dos coordinadoras de las asignaturas implicadas y les propuso cuatro profesores como posibles participantes en el proyecto de innovación docente. A continuación, tuvo lugar una primera reunión de la IP con los dos profesores de los GC en la que se les presentaron las líneas generales del proyecto de innovación docente y se tomaron decisiones conjuntas sobre su colaboración.

Teniendo en cuenta los resultados de la primera fase del proyecto al cual ya nos hemos referido, así como las características de los cuatro profesores participantes, las tres investigadoras introdujeron los ajustes necesarios en la propuesta de innovación docente inicial, concretando las estrategias y recursos que se introducirían en cada uno de los bloques temáticos, la evaluación, la información a los estudiantes, el documento de consentimiento para poder grabar en video algunas sesiones de clase y algunas discusiones en grupo en audio, etcétera. Durante el periodo de recogida de datos (septiembre-diciembre del curso 2015-2016), se trabajaron en la clase los tres primeros bloques temáticos del plan docente, de los seis que incluye.

En la segunda clase del curso se explicó a los estudiantes (más detalladamente en el caso de los GI) el proyecto, se pidió su consentimiento y se les proporcionó la dirección electrónica a la que debían enviar los registros en audio de las discusiones en grupo cooperativo en clase. A partir de la segunda clase del curso, comenzó la introducción de las innovaciones en los GI. Se llevaron a cabo tres registros en video de cada bloque (inicio-medio-final) de las dos profesoras de los GI (nueve en total). En el caso de los profesores del GC se registraron las sesiones de clase iniciales y finales de cada bloque (seis sesiones). Las tres investigadoras se reunieron de manera semanal desde el inicio de la recogida de datos para compartir el proceso de innovación, discutir las dudas y las dificultades, valorar los cambios e introducir reajustes. En general, se discutía con el fin de llegar a acuerdos respecto a la manera de gestionar las clases para conseguir que los estudiantes participasen en las clases realizando aportaciones a partir de sus experiencias personales como aprendices, como monitores, y en general, como personas que habían participado en 
situaciones educativas. Se discutían también estrategias para fomentar que los estudiantes valorasen la necesidad de leer los textos con atención para poder argumentar adecuadamente sus intervenciones.

\section{Procedimiento de análisis de datos}

Las clases han sido analizadas por una de las docentes que ha introducido innovaciones en sus clases, la investigadora (no docente) que participó en el diseño y seguimiento de la innovación docente, y tres estudiantes del grado de Psicología. Con el fin de acordar criterios de análisis de las actividades llevadas a cabo por los docentes y los estudiantes que participaron en el estudio, cada una de las estudiantes y las dos investigadoras analizaron de manera independiente cuatro sesiones de clase, una de cada uno de los docentes implicados, con cada uno de los dos instrumentos (rúbrica y EVALOE).

Después de llevar a cabo el análisis de una sesión de clase de manera independiente, se pusieron en común las puntuaciones en cada uno de los dos instrumentos de las dos investigadoras y las tres estudiantes, así como de las sesiones de clase entendidas globalmente (características de la sesión de clase, actividad que se propone, actitud general de los estudiantes y del profesor, estado de ánimo, etcétera), se discutieron los criterios seguidos, y se llevó a cabo el siguiente análisis de otra sesión y de otro docente.

Se procedió así hasta que los criterios fueron consensuados a través de un proceso de discusión y toma de acuerdos en el que se recorría un camino de ida y vuelta desde la valoración general cualitativa de las sesiones de clase, siguiendo los criterios que están en la base de la rúbrica y de la EVALOE, hasta las puntuaciones numéricas. Se llegó al consenso a través del análisis conjunto de sesiones de clase registradas en video. Cada una de las cinco componentes del equipo de análisis presentaba y argumentaba la manera como había discurrido la clase a partir de los dos instrumentos, con ejemplos concretos de las producciones de la docente y de los estudiantes, argumentando por qué había asociado una determinada puntuación en la rúbrica y en la EVALOE. Cuando se consideraba necesario se revisaba conjuntamente el video de la secuencia concreta de la clase que se estaba discutiendo y se aclaraban las posibles dudas sobre la manera de entender un indicador o alguno de los instrumentos de análisis. Una vez alcanzado este acuerdo, una de las estudiantes analizó las sesiones de clase de los dos docentes de los grupos de EP, y las otras dos estudiantes se repartieron el análisis de las sesiones de clase de los dos docentes de los grupos de EI.

\section{Resultados \\ Las discusiones entre las investigadoras durante el proceso}

Un primer resultado que nos interesa destacar es que las investigadoras se reunían de manera semanal para discutir las sesiones de clase, lo cual contribuía claramente a unificar la propuesta innovadora y a enriquecerla, puesto que se iban introduciendo elementos a partir de la reacción de las diferentes docentes y de los estudiantes a las propuestas realizadas en las clases, en la línea de lo que supone una investigación-acción.

En este sentido, el hecho de que los estudiantes de un grupo en particular señalasen que no estaban acostumbrados a leer los textos antes de iniciar las sesiones de clase, ni a participar antes de que las docentes explicasen en clase los contenidos de aprendizaje, suponía un reto no únicamente para la docente de este grupo, sino para la docente del otro grupo de innovación, que hacía propuestas que podían ser útiles para ambos grupos de alumnos.

De la misma manera, la participación de las estudiantes de grado en el análisis de los datos también contribuyó a incorporar la visión de los estudiantes en las discusiones sobre las propuestas de innovación y la manera como eran recibidas, interpretadas y vivenciadas por los estudiantes. 


\section{Las conversaciones en el grupo clase}

Se presentan los resultados del análisis de las sesiones de clase de EI en la tabla 3 y los de EP en la tabla 4. Como se aprecia, se han analizado las sesiones inicio y final de cada bloque, puesto que son las que se habían registrado en todos los grupos.

En la figura 1 se presentan los resultados totales obtenidos a partir del sumatorio de las puntuaciones obtenidas en las diferentes dimensiones de la rúbrica.

La primera pregunta de investigación que nos planteábamos al inicio era si se percibirían diferencias entre las sesiones de clase de los docentes que promoviesen de manera sistemática y planificada la participación oral de los estudiantes y aquellos que no lo hiciesen. Como se aprecia en las Tablas 3 y 4 y en la figura 1, en las sesiones de clase de los grupos de innovavión (GI) las puntuaciones son más altas que en las clases de los grupos de comparación (GC), y en el caso de los GI se incrementan a lo largo del periodo analizado, mientras que las clases de los GC hay pocas variaciones. Se observa que las docentes y los estudiantes de los GI incorporan el uso de gestos de diferente tipo y de expresiones faciales que acompañan al texto oral durante las conversaciones en clase, así como la entonación, especialmente por parte de los estudiantes, que son cada vez más conscientes de su importancia. Ello supone que las docentes han conseguido ayudar a los estudiantes a valorar la importancia de las expresiones faciales y de los gestos como recursos para conseguir captar la atención de sus compañeros y la de la docente, así como para apoyar sus argumentos verbales.

Tabla 3. Resultados del análisis de las sesiones de clase en los grupos de Educación Infantil*

\begin{tabular}{|c|c|c|c|c|c|c|c|c|c|c|c|c|}
\hline \multirow[b]{2}{*}{ Dimensiones } & \multicolumn{2}{|c|}{ Bloque 1} & \multicolumn{2}{|c|}{ Bloque 2} & \multicolumn{2}{|c|}{ Bloque 3} & \multicolumn{2}{|c|}{ Bloque 1} & \multicolumn{2}{|c|}{ Bloque 2} & \multicolumn{2}{|c|}{ Bloque 3} \\
\hline & I & $\boldsymbol{F}$ & I & $\boldsymbol{F}$ & I & $\boldsymbol{F}$ & I & $\boldsymbol{F}$ & I & $\boldsymbol{F}$ & I & $\boldsymbol{F}$ \\
\hline Multimodalidad & 1.5 & 2.0 & 3.0 & 3.0 & 3.0 & 3.0 & 1.0 & 1.0 & 1.0 & 1.0 & 1.0 & 1.0 \\
\hline Prosodia & 1.5 & 2.0 & 2.5 & 3.0 & 2.5 & 3.0 & 2.0 & 1.0 & 2.0 & 1.5 & 1.5 & 1.5 \\
\hline Estrategias argumentativas & 1.4 & 2.0 & 2.3 & 2.1 & 2.3 & 2.1 & 0.0 & 0.0 & 0.0 & 0.0 & 1.0 & 1.0 \\
\hline Léxico y terminología & 1.5 & 2.0 & 2.0 & 2.5 & 3.0 & 3.0 & 2.0 & 1.0 & 2.0 & 1.0 & 2.0 & 1.0 \\
\hline
\end{tabular}

* Se muestra la media en cada una de las siete dimensiones, puntuando del 1 al 4 en función del ajuste a la descripción cualitativa que se incluye en la rúbrica al inicio (I) y al final (F) de cada bloque temático. Se han puntuado con un 0 cuando una sesión de clase se ha considerado no evaluable en dicha dimensión. 
También se ponen de manifiesto avances en la gestión de la conversación, especialmente por el hecho de que los estudiantes hacen referencia a las intervenciones anteriores de sus compañeros cuando toman la palabra e incorporan progresivamente estrategias de cortesía, que modela la docente. Cabe señalar que la incorporación de las estrategias argumentativas es más lenta por la complejidad que supone a menudo para los estudiantes aportar razones claras a sus intervenciones, a pesar de las ayudas que proporciona la docente, aunque éstas supongan a menudo hacerles conscientes de la importancia de referirse a las intervenciones previas de los compañeros, así como a la necesidad de estar atentos a los gestos o entonación para tenerlos en cuenta en sus contribuciones posteriores, en el sentido que consideren oportuno.

\section{Figura 1. Resultados totales de la evaluación de las sesiones de clase en los grupos de EI}

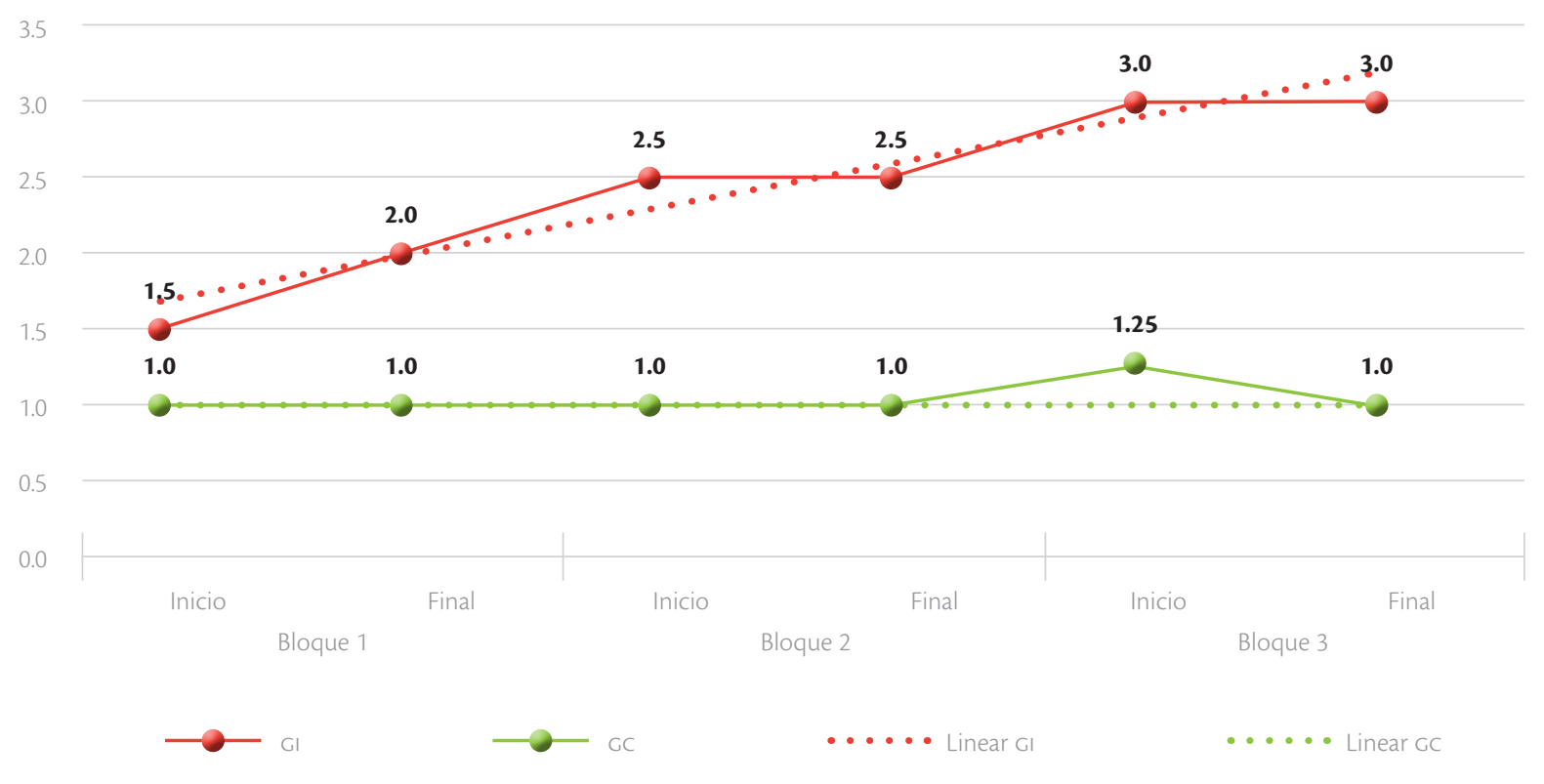

El análisis global de las sesiones de los cuatro grupos pone de relieve que en las clases de los GI los estudiantes participan con mucha más frecuencia durante toda la sesión que los estudiantes de los GC, puesto que las docentes de los GI promueven que lo hagan usando estrategias diversas, algunas de las cuales se van a revisar más adelante, como son la explicitación de los conocimientos previos o de los objetivos de aprendizaje en relación con la lengua oral.

La segunda pregunta de investigación se centraba en las diferencias entre las sesiones de clase en función del momento dentro de la unidad temática, es decir, la diferencia entre las clases de inicio de bloque y final en un mismo grupo clase. En las tablas 3 y 4 y en la figura 1 se aprecia que en el caso de los GI no hay diferencias en este sentido y en cambio en los GC se aprecia un ligero incremento de las puntuaciones en las sesiones iniciales. El análisis cualitativo global de los videos de las sesiones de clase realizado conjuntamente por las cinco personas que han participado en el análisis de los datos, tomando como base la estructura de los instrumentos de análisis, ha permitido detectar que el docente del GC de EI planteaba la sesión inicial de clase como una revisión de 
conocimientos previos, lo cual promovía mayor participación de los estudiantes. En cambio, en la clase final, el protagonismo era del profesor, quien resumía los contenidos principales del bloque trabajado. En esta última sesión se hacía evidente que el docente no incluía a los estudiantes en la síntesis final del bloque, y no recuperaba conocimientos previos iniciales con el fin de hacerles ver el proceso de construcción que habían seguido durante el trabajo del bloque a través de la lengua oral, estrategia que sí utilizaban las dos docentes de los grupos de innovación.

En análisis cualitativo de los videos permite detectar que el docente del GC de EI dedicó la primera sesión observada a revisar conocimientos previos y a debatir sobre cuestiones fundamentales de la materia, lo cual propició mucha participación por parte de los estudiantes. Esta metodología se dejó de usar en las siguientes clases, incluso en aquellas en las que se iniciaba un bloque temático, aunque sí se promovía más la participación de los estudiantes, lo cual se refleja en las observaciones realizadas en las sesiones iniciales de los bloques 2 y 3 .

En la tabla 4 se presentan los resultados de las sesiones de clase vinculados a los grupos de educación primaria. En la figura 2 se presentan los resultados totales obtenidos a partir de la sumatoria de las puntuaciones obtenidas en las diferentes dimensiones de la rúbrica.

De la misma manera que en los grupos de EI, en el caso de EP se pone de relieve una diferencia importante entre el GI, en el que la docente planifica de manera sistemática la incorporación en sus clases de estrategias argumentativas, de gestión de la conversación, entre otras, en sus clases y el GC.

Tabla 4. Resultados de la evaluación de las sesiones de clase en los grupos de Educación Primaria*

\begin{tabular}{|c|c|c|c|c|c|c|c|c|c|c|c|c|}
\hline \multirow[b]{3}{*}{ Dimensiones } & \multicolumn{6}{|c|}{ Grupo de Innovación (GI) } & \multicolumn{6}{|c|}{ Grupo de Comparación (GC) } \\
\hline & \multicolumn{2}{|c|}{ Bloque 1} & \multicolumn{2}{|c|}{ Bloque 2} & \multicolumn{2}{|c|}{ Bloque 3} & \multicolumn{2}{|c|}{ Bloque 1} & \multicolumn{2}{|c|}{ Bloque 2} & \multicolumn{2}{|c|}{ Bloque 3} \\
\hline & I & $\boldsymbol{F}$ & I & $\boldsymbol{F}$ & I & $\boldsymbol{F}$ & I & $\boldsymbol{F}$ & I & $\boldsymbol{F}$ & I & $\boldsymbol{F}$ \\
\hline Multimodalidad & 2.3 & 2.5 & 2.8 & 2.8 & 2.8 & 2.8 & 2.0 & 0.0 & 1.8 & 2.0 & 2.5 & 2.5 \\
\hline Prosodia & 3.0 & 3.0 & 3.0 & 2.5 & 3.0 & 3.0 & 2.5 & 1.0 & 1.5 & 2.0 & 3.0 & 2.0 \\
\hline Estrategias argumentativas & 1.7 & 2.0 & 2.1 & 2.3 & 2.1 & 2.4 & 1.3 & 0.1 & 0.0 & 1.7 & 1.4 & 1.3 \\
\hline Léxico y terminología & 2.0 & 2.0 & 3.0 & 2.5 & 2.0 & 3.0 & 1.0 & 0.0 & 0.5 & 2.0 & 1.5 & 2.0 \\
\hline
\end{tabular}

* Se muestra la media en cada una de las siete dimensiones, puntuando del 1 al 4 en función del ajuste a la descripción cualitativa que se incluye en la rúbrica al inicio (I) y al final ( $F$ ) de cada bloque temático. Se han puntuado con un 0 cuando una sesión de clase se ha considerado no evaluable en dicha dimensión. 
Figura 2. Resultados totales de la evaluación de las sesiones de clase en los grupos de EP

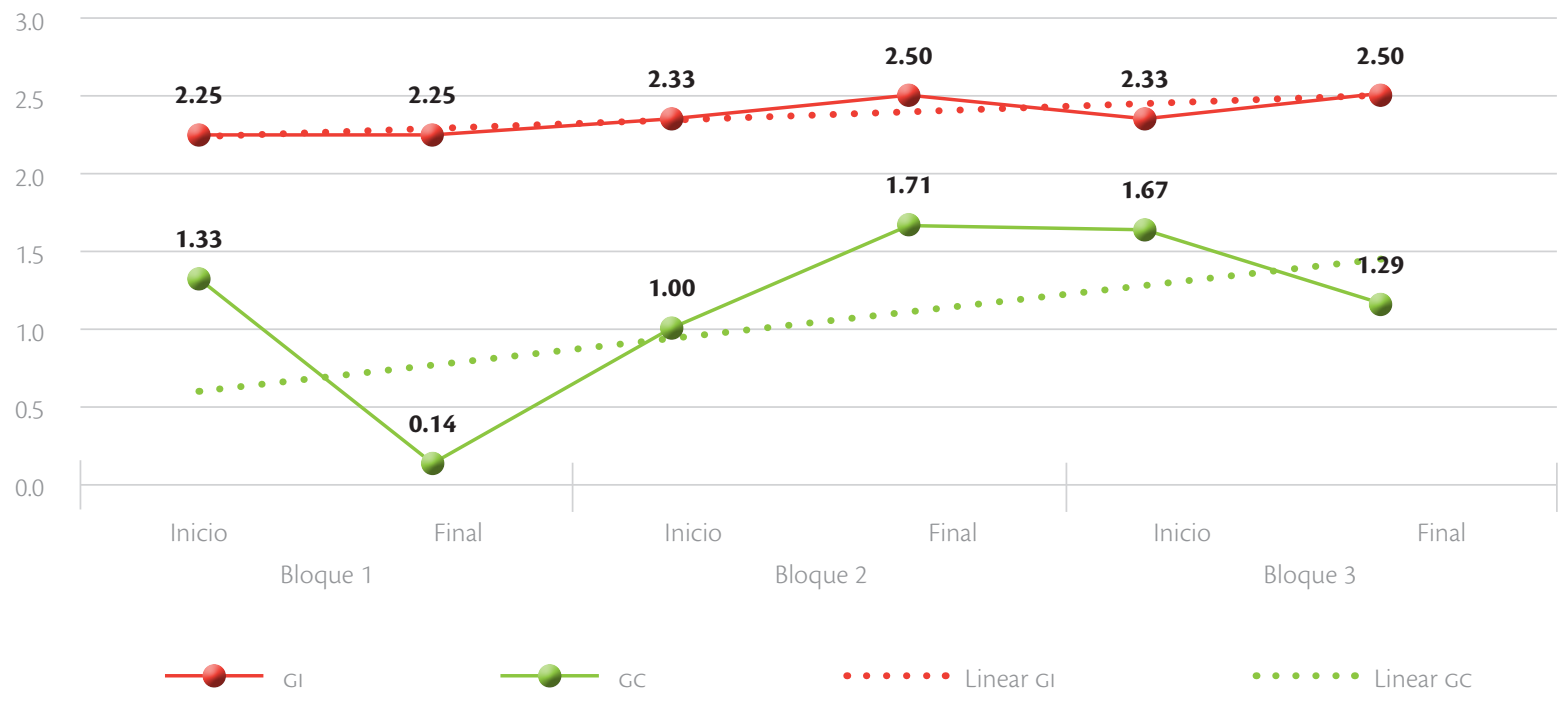

Las tablas 5 y 6 y las Figuras 3 y 4 muestran los resultados relativos al análisis de las sesiones de clase con la EVALOE en EI y EP, respectivamente. Los resultados evidencian diferencias importantes entre las sesiones de clase de los dos GI y de los dos GC, tanto respecto a cada una de las tres dimensiones de la EVALOE como en el total de la escala. Los resultados son más evidentes en el GI de EI, puesto que, además de la diferencia inicial respecto al GC, manifiestan la incorporación de estrategias discursivas y conversacionales a lo largo del trabajo de los tres bloques temáticos.

Tabla 5. Resultados de la evaluación de las sesiones de clase con EVALOE en los grupos de EI*

\begin{tabular}{|c|c|c|c|c|c|c|c|c|c|c|c|c|}
\hline \multirow[b]{3}{*}{ Subescalas } & \multicolumn{6}{|c|}{ Grupo de Innovación (GI) } & \multicolumn{6}{|c|}{ Grupo de Comparación (GC) } \\
\hline & \multicolumn{2}{|c|}{ Bloque 1} & \multicolumn{2}{|c|}{ Bloque 2} & \multicolumn{2}{|c|}{ Bloque 3} & \multicolumn{2}{|c|}{ Bloque 1} & \multicolumn{2}{|c|}{ Bloque 2} & \multicolumn{2}{|c|}{ Bloque 3} \\
\hline & I & $\boldsymbol{F}$ & I & $\boldsymbol{F}$ & $I$ & $\boldsymbol{F}$ & $\boldsymbol{I}$ & $\boldsymbol{F}$ & $I$ & $\boldsymbol{F}$ & $\boldsymbol{I}$ & $\boldsymbol{F}$ \\
\hline $\begin{array}{l}\text { Contexto y gestión de la } \\
\text { comunicación }\end{array}$ & $71 \%$ & $71 \%$ & $88 \%$ & $79 \%$ & $88 \%$ & $83 \%$ & $54 \%$ & $33 \%$ & $54 \%$ & $33 \%$ & $50 \%$ & $33 \%$ \\
\hline Diseño instruccional & $62 \%$ & $57 \%$ & $67 \%$ & $62 \%$ & $67 \%$ & $62 \%$ & $33 \%$ & $38 \%$ & $33 \%$ & $38 \%$ & $33 \%$ & $38 \%$ \\
\hline $\begin{array}{l}\text { Funciones comunicativas y } \\
\text { estrategias }\end{array}$ & $58 \%$ & $58 \%$ & $71 \%$ & $67 \%$ & $71 \%$ & $67 \%$ & $38 \%$ & $38 \%$ & $38 \%$ & $38 \%$ & $38 \%$ & $38 \%$ \\
\hline
\end{tabular}

* Se muestra el nombre de las tres subescalas y porcentaje alcanzado en cada sesión respecto al que se podría haber alcanzado (100\%). 
En la figura 3 se presentan los resultados totales de la evaluación de las clases con EVALOE en los grupos de EI. En la tabla 6 y la figura 3 se presentan los resultados obtenidos a partir del análisis de los dos grupos de EP con la EVALOE, por dimensiones y totales, respectivamente.

Los análisis de las sesiones de clase llevados a cabo con el instrumento de observación EVALOE ponen de relieve que las docentes de los GI introducen en sus clases más estrategias dirigidas a promover que los estudiantes participen oralmente en las clases, que gestionen la conversación y que sean más conscientes de que están usando la lengua oral para aprender y para mejorarla, que los docentes de los GC. En el caso del GI de EI, se observa un incremento de estas estrategias y habilidades por parte de los estudiantes a lo largo del periodo de recogida de datos más evidente que en las clases del GI de EP. En general, se muestra que la docente y los estudiantes del GI de EI explicitan más en sus clases las estrategias que están usando, lo que explica las puntuaciones más elevadas en la subescala de Diseño instruccional.

Figura 3. Resultados totales de la evaluación de las sesiones de clase con EVALOE en los grupos de EI

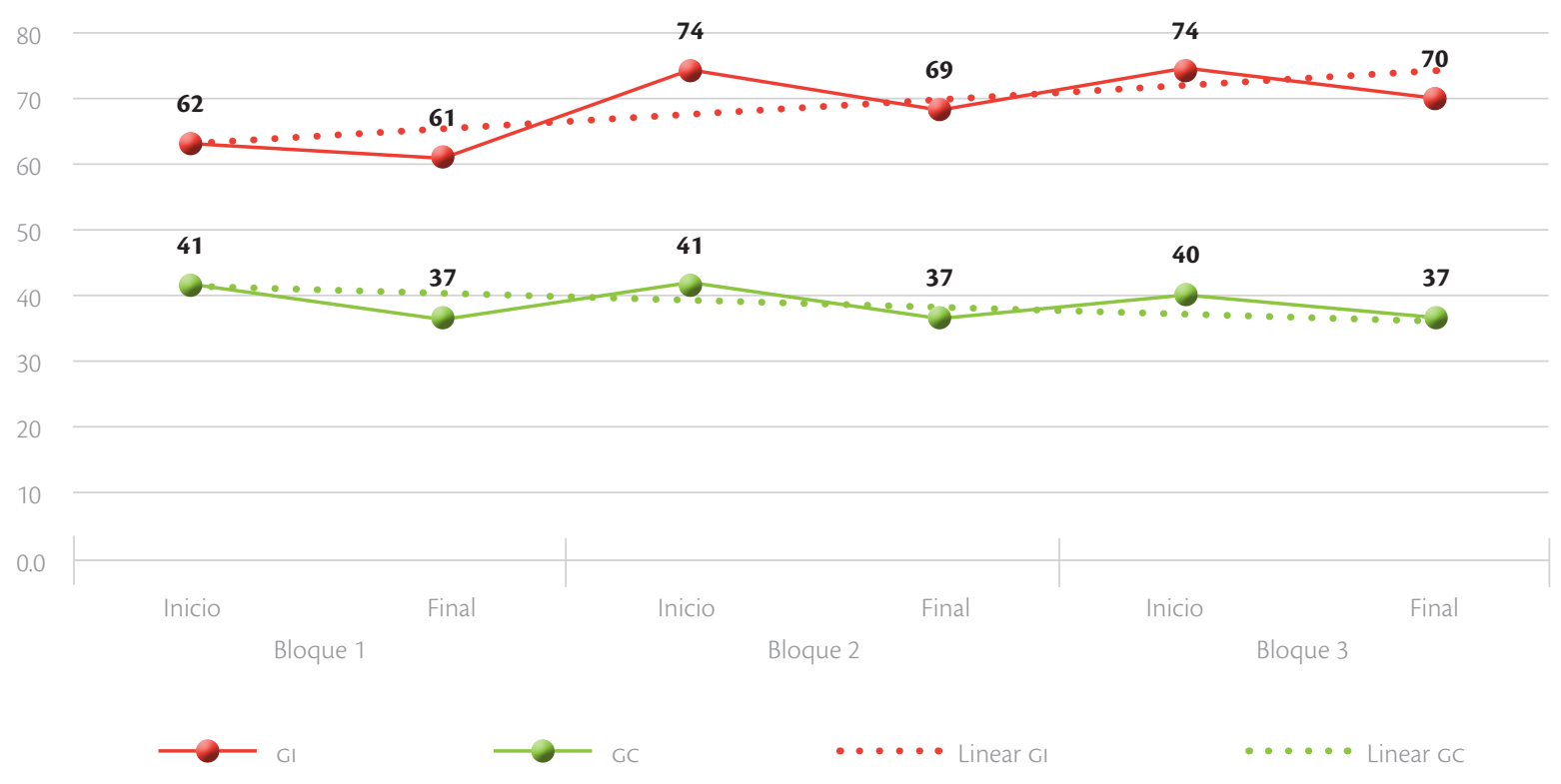

Nota: se muestra el porcentaje alcanzado en el inicio y el final de cada bloque respecto al que se podría haber alcanzado (100\%). 


\section{Figura 4. Resultados totales de la evaluación de las sesiones de clase con EVALOE} en los grupos de EP

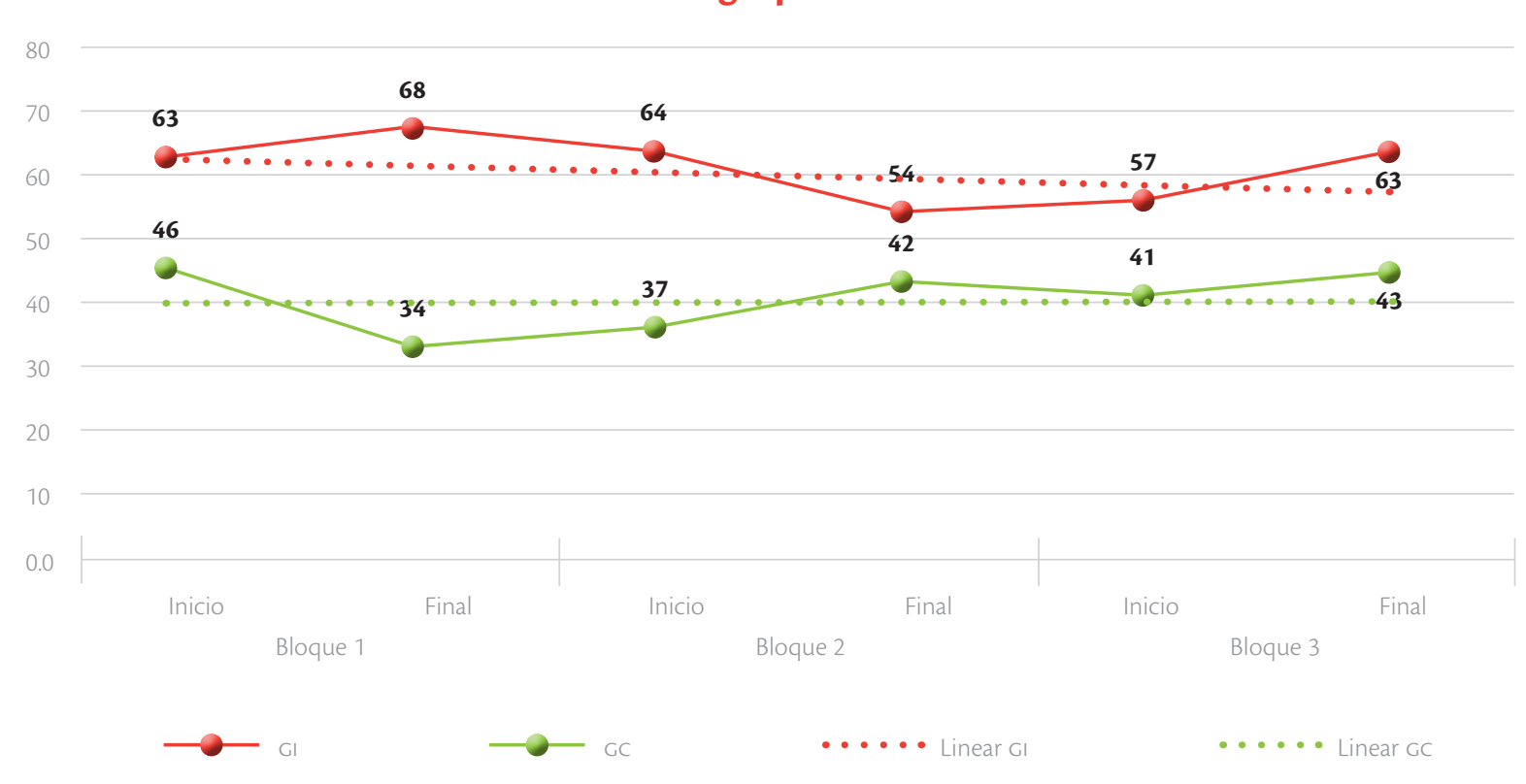

Nota: se muestra el porcentaje alcanzado en el inicio y el final de cada bloque respecto al que se podría haber alcanzado (100\%).

\section{Las discusiones en pequeño grupo en clase}

En la figura 5 se presentan los resultados de los GI y GC de EI relativos al análisis de las discusiones en pequeño grupo. Los resultados del GI indican que, a partir de unas puntuaciones relativamente bajas, alrededor del 1.5 sobre 4 , en las dos medidas tomadas durante el trabajo de los bloques 2 y 3 , las puntuaciones se incrementan claramente, lo cual significa que los estudiantes incorporan las estrategias conversacionales y argumentativas también durante el trabajo en pequeño grupo. En cambio, las puntuaciones de los estudiantes de GC disminuyen ligeramente respecto a la primera medida, durante el trabajo del bloque 1.

En la figura 6 se pueden apreciar las dimensiones que mejoran a lo largo del trabajo de los tres bloques de la asignatura en un único grupo pequeño de trabajo del GI. En la figura 7 se presenta la evolución de tres grupos de trabajo del GI de EP a lo largo del trabajo de los tres bloques temáticos.

Finalmente, la figura 8 permite detectar el comportamiento específico de las estrategias concretas que recoge el instrumento de análisis. Se aprecia cómo algunas de las habilidades, por ejemplo aquellas vinculadas a la prosodia, muy pronto obtienen una puntuación elevada, sin alcanzar la puntuación más alta (4). En cambio, otras habilidades como el uso de recursos que contribuyen a la cohesión textual o las estrategias argumentativas, parten de una puntuación inicial muy baja, que se incrementa progresivamente, sin alcanzar una puntuación más elevada del 2.5.

\section{Discusión de los resultados}

Este artículo explora los cambios en las características de la interacción y de los textos orales construidos por los docentes y los estudiantes en las sesiones de clase cuando se introducen instrumentos, recursos y estrategias por parte de docentes universitarios con el fin de incrementar la participación oral de los estudiantes universitarios y contribuir a su competencia comunicativa y lingüística.

Las observaciones realizadas muestran que la incorporación progresiva en las clases, a lo largo del trabajo de los tres bloques de la asignatura, de los elementos vinculados a la conversación argumentativa, 
Figura 5. Resultados de la evaluación de las sesiones de dos grupos pequeños de EI

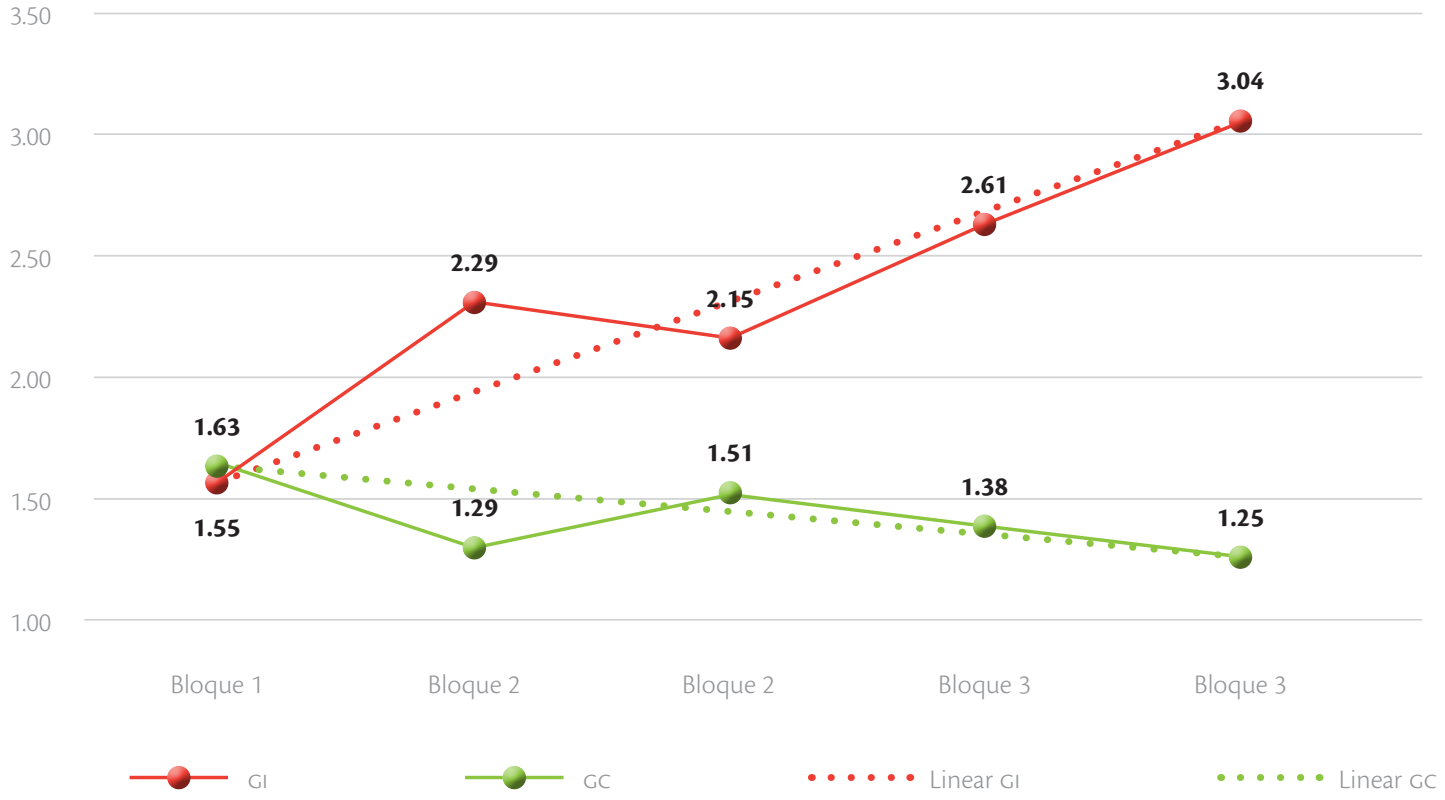

Nota: se muestran las medias de las evaluaciones en cada bloque, puntuando del 1 al 4 en función del ajuste a la descripción cualitativa que se incluye en la rúbrica.

Figura 6. Resultados de la evaluación de las sesiones de un solo grupo pequeño de EI

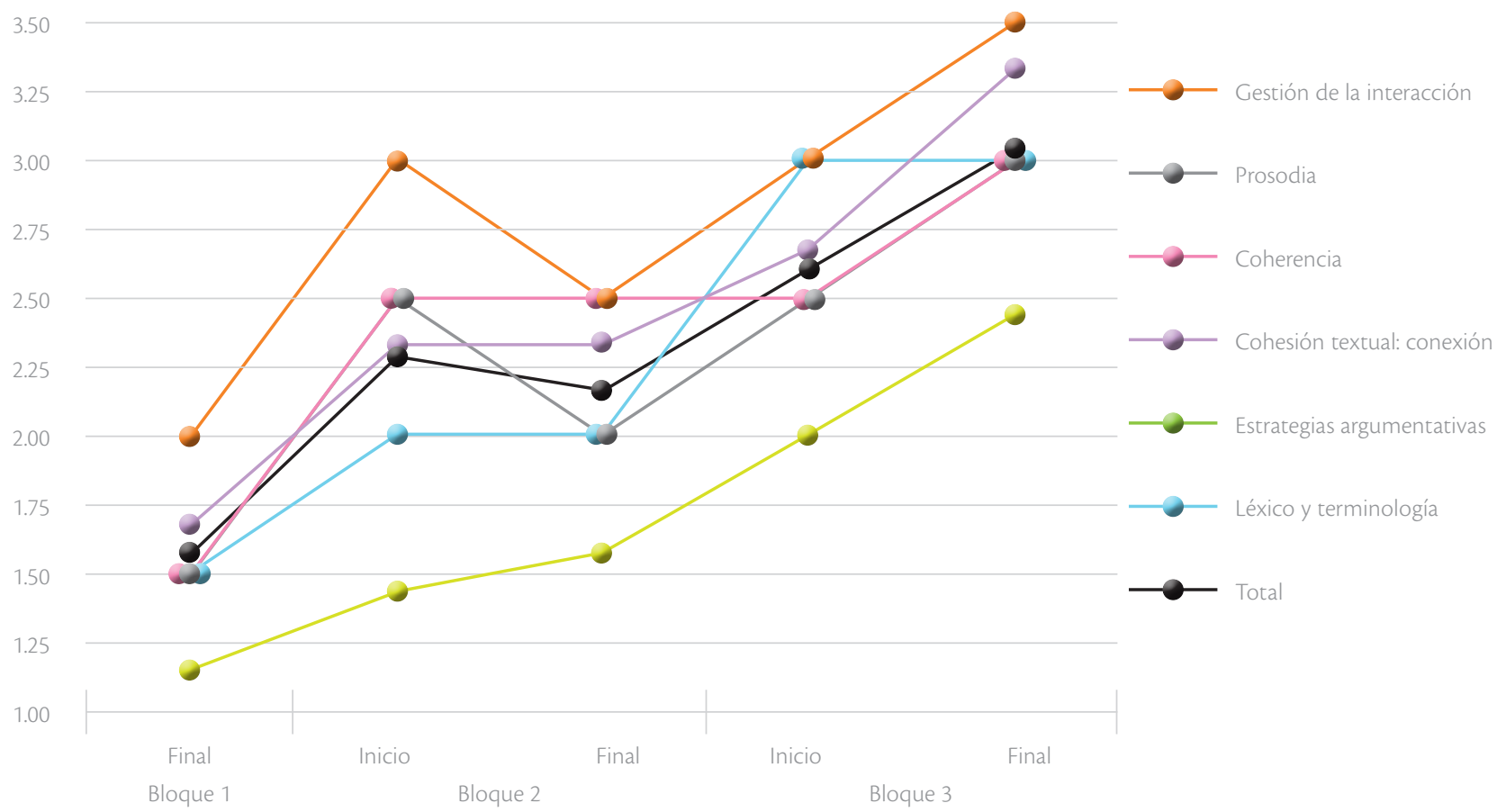


Figura 7. Resultados de la evaluación de las sesiones de tres grupos pequeños de EP

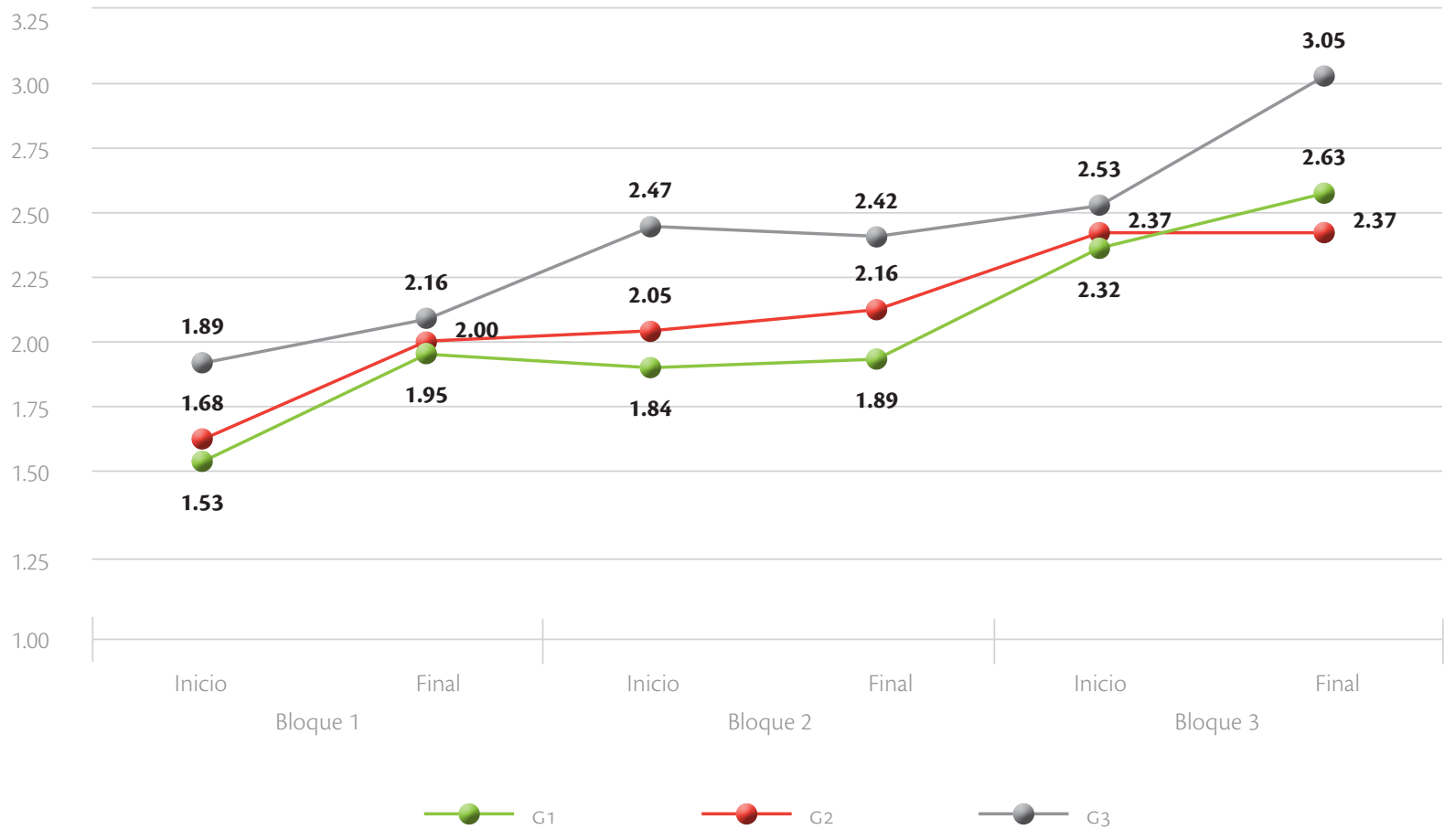

Nota: se muestran las medias de las evaluaciones en cada bloque, puntuando del 1 al 4 en función del ajuste a la descripción cualitativa que se incluye en la rúbrica.

Figura 8. Resultados de la evaluación de las sesiones de un solo grupo pequeño de EP

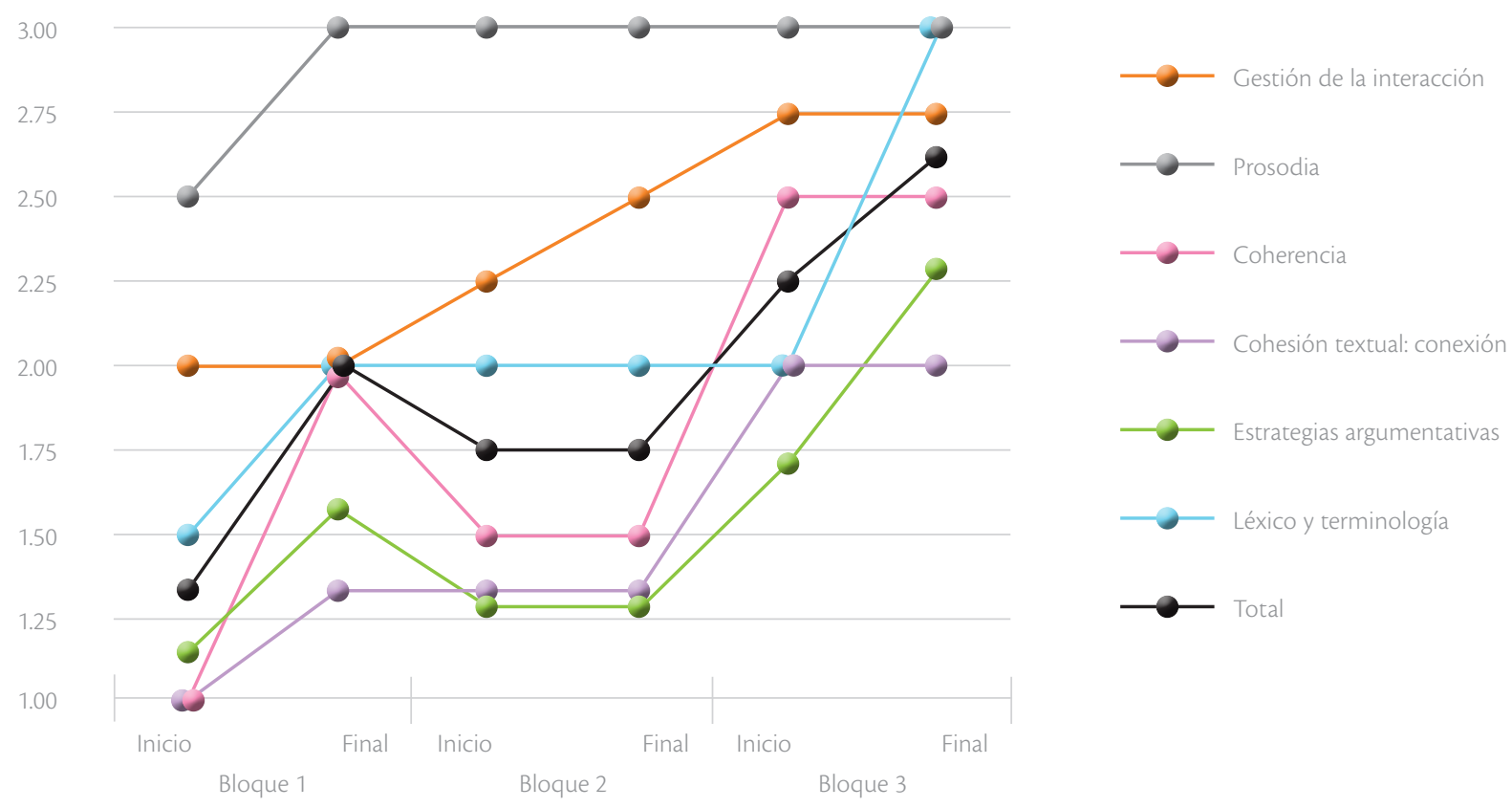


tanto en el GI de EI como en el GI de EP, promueve una mayor participación oral de los estudiantes universitarios, así como una mayor consciencia sobre su aprendizaje. La observación de estos cambios parece indicar que la introducción progresiva de elementos, instrumentos y momentos de reflexión sobre las discusiones argumentativas en las clases por parte de los docentes de los GI ha sido incorporada en las clases por parte de los estudiantes, como ha venido siendo reclamado por voces diversas respecto a la formación de maestros (Ogienko y Rolyak, 2009; Cano, 2010; Doherty et al., 2011; Monarca, 2013) y en el sentido que proponen diversos autores (Felton $e t$ al., 2015; Leitão, 2011, entre otros).

Si nos centramos en los datos que nos proporciona la escala EVALOE, se pone de manifiesto que desde el inicio de la asignatura las docentes introducen cambios en las clases que promueven la mayor participación de los estudiantes, que en uno de los grupos se incrementa a lo largo del semestre. En cambio, en el caso de los docentes de los GC, las características de las clases se mantienen durante el trabajo de los tres bloques de contenido.

Si revisamos los baremos orientativos que establece el manual de la escala de observación EVALOE (Gràcia et al., 2015a) para los niveles de educación infantil y primaria, las docentes de los GI se situarían en la franja media y los docentes de los GC se situarían en la franja baja. Se trata de unos baremos establecidos para otro tipo de situaciones de educación formal, muy diferentes de los que estamos analizando en este estudio. Aun así, nos pueden estar indicando que, a pesar de que en los GC se han incorporado estrategias por parte de docentes y de estudiantes, es posible incorporar más elementos que indicarían que los estudiantes son capaces de gestionar la conversación en mayor medida (Schegloff, 2007), que para ello utilizan recursos prosódicos (Selting, 2010) y multimodales (Müller et al., 2013, 2014), que las actividades de discusión tienen un formato en red, que los estudiantes incorporan la autoevaluación de su participación como algo habitual en las clases (Kuhn et al., 2013) o que utilizan el lenguaje con intenciones comunicativas diversas y todo ello contribuye a su aprendizaje activo (Leitão, 2000; Lefstein y Snell, 2014) y a un compromiso con el entorno inmediato (Monarca, 2013).

En conjunto, los resultados parecen señalar que la innovación diseñada y aplicada para mejorar la competencia comunicativa y lingüística oral de los estudiantes de FIM a través de los instrumentos elaborados e incorporados en las sesiones del primer semestre de clase (septiembre-diciembre) en una asignatura anual de primer curso, ha permitido observar cambios progresivos en la manera de actuar de los docentes y de los estudiantes en el sentido que se pretendía.

Las clases de los GI incorporan elementos vinculados a la Metodología conversacional, esto es, una manera de entender la docencia como una actividad participativa, activa, que parte del cuestionamiento, de la revisión conjunta de los conocimientos previos, del planteamiento de problemas que es necesario discutir y llegar a acuerdos mediante la presentación y exposición de argumentos y contrargumentos, contribuyendo al desarrollo de la competencia argumentativa y fomentando el pensamiento crítico (Felton et al., 2015; Lefstein y Snell, 2014; Leitão, 2011; Ogienko y Rolyak, 2009; van Eemeren et al., 2002; Walton, 2013).

$\mathrm{Al}$ mismo tiempo, este tipo de situaciones se aleja de las clases universitarias tradicionales en las que el conocimiento parece estar únicamente en manos del profesor, que tiene como labor transmitirlo a los estudiantes y éstos reproducirlo en el momento de la evaluación (Pozo, 2016).

En síntesis, la particular aportación de este estudio reside en el hecho de dotar a los alumnos de autonomía en la reflexión y toma de conciencia de los recursos linguísticos e interactivos que posibilitan y contribuyen a la calidad de estos procesos dialógicos para el aprendizaje. Sin un buen dominio de estos recursos, conceptualizados como herramientas semióticas que 
se pueden aprender mediante su uso en contextos funcionales, no es posible un diálogo igualitario entre los

\section{Referencias}

Acosta, F. M. (2012), "Educar, enseñar, escolarizar: el problema de la especificación en el devenir de la Pedagogía (y la transmisión)", Tendencias Pedagógicas, núm. 20, pp. 93-105.

Blair, J. A. y R. H. Johnson (1987), "Argumentation as dialectical", Argumentation, vol. 1, núm. 1, pp. 41-56.

Bublitz, W. (2011), "Cohesion and coherence", en Discursive pragmatics, Amsterdam, John Benjamins, pp. 37-49.

Burke, J. et al. (2007), "Toward a Definition of Mixed Methods Research", fournal of Mixed Methods Research, vol. 1, núm. 2, pp. 112-133.

Cabré, M. T. (1999), La terminología. Representación y comunicación, Barcelona, Institut Universitari de Lingüística Aplicada.

Calsamiglia, H. y A. Tusón (1999), Las cosas del decir, Barcelona, Ariel.

Cano, M. I. (2010), "Argumentació y construcció del coneixement: Estratègies argumentatives dels estudiants universitaris en situació de debat", tesis doctoral, Barcelona, Facultat de Psicologia, Ciències de l'Educació i de l'Esport Blanquerna, Universitat Ramon Llull.

Charaudeau, P. y D. Maingueneau (2002), Dictionnaire d'analyse du discours, París, Editions du Seuil [Trad. Esp.: Diccionario de análisis del discurso, Buenos Aires, Amorrortu, 2005].

Coll, C. (2013), "La educación formal en la nueva ecología del aprendizaje: tendencias, retos y agenda de investigación”, en J. L. Rodríguez Illera (coord.), Aprendizaje y educación en la sociedad digital, Barcelona, Universitat de Barcelona, pp. 156-170 [DOI: 10.1344/106.000002060].

Cruse, A. (2011), Meaning in language: an introduction to semantics and pragmatics, Oxford, Oxford University Press.

Cuenca, M. J. (2007), "Modalització i text argumentatiu”, Articles, núm. 42, pp. 33-43. diversos participantes (docente y aprendices) que sustente el proceso de construcción social intersubjetiva.
Cuenca, M. J. (1995), "Mecanismos lingüísticos y discursivos de la argumentación”, Comunicación, Lenguaje y Educación, núm. 25, pp. 23-40.

Doherty, C. A. et al. (2011), "Talking the talk: oracy demands in first year university assessment tasks", Assessment in Education, vol. 18, núm. 1, pp. 27-39.

Felton, M. et al. (2015), "Arguing collaboratively: Argumentative discourse types and their potential for knowledge building", British fournal of Educational Psychology, núm. 85, pp. 372-386.

Firth, J. R. (1957), Papers in linguistics (1934-1951), Londres, Oxford University Press.

Generalitat de Catalunya (2013), Competències bàsiques de l'àmbit lingüistic. Llengua catalana $i$ llengua castellana. Identificació $i$ desplegament a l'educació primària [Competencias básicas del ámbito lingüístico. Catalán y español. Identificación y despliegue en la educación primaria], Barcelona, Generalitat de Catalunya.

Gràcia, M. et al. (2017), "Análisis de las líneas de investigación y actuación en la enseñanza y el aprendizaje del lenguaje oral en contexto escolar", Revista Española de Linguística Aplicada, vol. 30, núm. 1, pp. 188-209.

Gràcia, M. et al. (2016), "Construcción y reflexión metalingüística de textos orales monogestionados y plurigestionados en el grado de maestro. Estudio piloto", Revista del Congrés Internacional de Docència Universitària i Innovació (CIDUI), 3, <http://www. cidui.org/revistacidui/index.php/cidui/article/ view/842>.

Gràcia, M. et al. (2015a), Evaluación de la enseñanza de la lengua oral. Escala EVALOE, Barcelona, Graó.

Gràcia, M. et al. (2015b), "Developing and testing EVALOE: a tool for assessing spoken language teaching and learning in the classroom", Child Language Teaching and Therapy, vol. 31, núm. 3, pp. 287-304. 
Gràcia, M. et al. (2015c), "Propuestas de innovación de la enseñanza de la competencia comunicativa y discursiva en la formación universitaria de futuros maestros", Actes del V Congrés Internacional UNIVEST 2015. Els reptes de millorar l'avaluació, Girona, pp. 449-453, <http:// www.udg.edu/Portals/9/Publicacions/electroniques/ Univest.pdf> [Consulta: octubre de 2017]. Gràcia, M. et al. (2015d), "La competencia lingüística (hablar y escuchar) en el grado de Maestro. Propuestas de mejora mediante la metodología conversacional y el trabajo colaborativo", en Presente y futuro de la docencia universitaria, Ourense, Educación Editora, pp. 171-176.

Gràcia, M. et al. (2012), "El cambio conceptual de dos maestras en relación con la enseñanza y el aprendizaje de la lengua oral a través de un asesoramiento: un estudio de casos", Revista de Logopedia, Foniatría y Audiología, vol. 32, núm. 4, pp. 179-189.

Halliday, M. A.K. y Hasan, R. (1976), Cohesion in English, Londres, Longman.

Hoffman, J. L. (2011), "Coconstructing meaning: interactive literary discussions in kindergarten readalouds", The Reading Teacher, vol. 65, núm. 3, pp. 183-194.

Ishihara, N. y A. D. Cohen (2014), Teaching and learning pragmatics: where language and culture meet, Londres, Routledge.

Justice, L.M. y H. K. Ezell (1999), "Vygotskian theory and its application to language assessment: An overview for speech-language pathologists", Contemporary Issues in Communication Science and Disorders, núm. 26, pp. 111-118.

Kathard, H. et al. (2015), "A study of teacher-learner interactions: a continuum between monologic and dialogic interactions", Language, speech, and hearing services in schools, vol. 46, núm. 3, pp. 222-241.

Kuhn, D. et al. (2013), "Developing norms of argumentation: metacognitive, epistemological, and social dimensions of developing argumentative competence", Cognition E̊ Instruction, núm. 31, pp. 456-496.

Kuhn, D. (1991), The skills of argument, Cambridge, Cambridge University Press.

Latorre, A. (2003), La investigación-acción: conocer y cambiar la práctica educativa, Barcelona, Graó.
Lefstein, A. yJ. Snell (2014), Better than best practice. Developing teaching and learning through dialogue, Abingdon, Routledge.

Leitão, S. (2000), "The potential of argument in knowledge Building”, Human Development, núm. 43, pp. 332-360.

Leitão, S. (201 1), "O lugar da argumentação na construcção do conhecimento em sala de aula", en Argumentação na escola: o conhecimento em construção, Campinas, Pontes Editores, pp. 13-46.

Li, M. et al. (2015), "Exploring the nature of teacherstudent interaction in small-group discussions in a Chinese university setting", fournal of Computers in Education, vol. 2, núm. 4, pp. 475-491.

Lo Cascio, V. (1998), Gramática de la argumentación, Madrid, Alianza.

Marinac, J. et al. (2004), "Talking to children: The withinclassroom nature of everyday adult language input in the early childhood education environment", International Fournal of Speech-Language Pathology, vol. 6, núm. 4, pp. 237-246.

Mercer, N. (2010), "The analysis of the classroom talk: Methods and methodologies", British fournal of Educational Psychology, núm. 1, pp. 1-14.

Monarca, H. (2013), "Participación dialógica en la universidad: condición para el desarrollo del pensamiento crítico y el compromiso social", Revista Iberoamericana de Educación Superior (RIES), Vol. IV, núm. 9, pp. 53-62, <http://ries.universia.net/index. $\mathrm{php} / \mathrm{ries} /$ article/view/305/html_40> [Consulta: noviembre de 2017].

Mondada, L. (2013), "Multimodal interaction", en G. Müller et al. (eds.), Body-Language-Communication. An International Handbook on Multimodality in Human Interaction, Berlín, De Gruyter Mouton, pp. 577-589.

Müller, C. et al. (eds.) (2014), Body-Language-Communication.

Volume 2: An International Handbook on Multimodality in Human Interaction, Berlín, De Gruyter Mouton.

Müller, C. et al. (eds.) (2013), Body-Language-Communication. Volume 1: An International Handbook on Multimodality in Human Interaction, Berlín, De Gruyter Mouton.

Ogienko, O. y A. Rolyak (2009), "Model of professional teachers' competences formation: European 
dimension", Proceedings TEPE 3rd Annual Conference. TEacher Education Policy in Europe: Quality in Teacher Education, Umea, Teacher Education Policy in Europe (TEPE) Network, pp. 158-168. <https:// tepe.files.wordpress.com/2010/02/tepe_conference_ proceedings09.pdf> [Consulta: noviembre de 2017].

Payrató, L. (2013), "Gestures in Southwest Europe: Catalonia", en C. Müller et al. (eds.), Body-LanguageCommunication. An International Handbook on Multimodality in Human Interaction, Berlín, De Gruyter Mouton, vol. 2, pp. 1266-1270.

Payrató, L. y S. Teßendorf (2014), "Pragmatic gestures", en C. Müller et al. (eds.), Body-Language-Communication. An International Handbook on Multimodality in Human Interaction, Berlín, De Gruyter Mouton, vol. 2, pp. 1531 1549.

Peña, L. B. (2008), "La competencia oral y escrita en la educación superior", en <http://www.mineducacion. gov.co/1621/articles-189357_archivo_pdf_ comunicacion.pdf> [Consulta: noviembre de 2017].

Perelman, C. y L. Olbrechts-Tyteca ([1958]1989), Tratado de la argumentación: la nueva retórica, Madrid, Gredos.

Pertusa, E. y Jarque, M. J. (2015), Estratègies per a la millora de la competència escrita, Barcelona, Universitat de Barcelona.

Pozo, J. I. (2016), Aprender en tiempos revueltos, Madrid, Alianza.

Rapanta, C. y D. Walton (2016), "Identifying paralogisms in two ethnically diferent contexts at university level", Infancia y aprendizaje, vol. 39, núm. 1, pp. 119-149.

Renkema,J.(2004),Introduction to discoursestudies, Ámsterdam, John Benjamins.

Riba, C. (2009), El proceso de investigación científica, Barcelona, Universitat Oberta de Catalunya.

Ruiz-Muñoz, M.J. (2012), "El desarrollo de competencias orales y escritas en el marco del Espacio Europeo de Educación Superior (EEES). Reflexiones, propuestas y experiencias en el grado en publicidad y relaciones públicas", Revista de Comunicación Vivat Academia, vol. XIV, pp. 133-144.

Selting, M. (2010), "Prosody in interaction: state of the art", en Prosody in Interaction, Amsterdam, John Benjamins, pp. 3-40.

Schegloff, E. A. (2015), "Conversational interaction: the embodiment of human sociality", en D. Tannen, H. E. Hamilton y D. Schiffrin (eds.), The Handbook of Discourse Analysis, Hoboken, NJ, John Wiley \& Sons, pp. 346-366.

Schegloff, E. A. (2007), Sequence organization in interaction. A primer in conversation analysis, Cambridge, Cambridge University Press.

Swain, M. y S. Lapkin (2013), "A Vygotskian sociocultural perspective on immersion education: The L1/L2 debate", Journal of immersion and content-based Language education, vol. 1, núm. 1, pp. 101-129.

Toulmin, S. E. (1958), The uses of argument, Cambridge, Cambridge University Press.

Van Dijk, T. A. (1983), La ciencia del texto. Un enfoque interdisciplinario, Barcelona, Paidós.

Van Eemeren, F. H. et al. (2002), Argumentation: analysis, evaluation, presentation, Mahwah, NJ, Lawrence Erlbaum.

Verschueren, J. (1999), Understanding pragmatics, Londres, Arnold.

Walton, D. N. (2013), Methods of argumentation, Cambridge, Cambridge University Press.

Walton, D. N. (2006), Fundamentals of critical argumentation, Nueva York, Cambridge University Press.

Weinstock, M. et al. (2004), "Missing the point or missing the norms? Epistemological norms as predictors of students' ability to identify fallacious arguments", Contemporary Educational Psychology, núm. 29, pp. 77-94.

Zlatić, L. et al. (2014), "Development of teacher communicative competence", Social and Behavioral Sciences, núm. 116, pp. 606-610.

\section{Cómo citar este artículo:}

Gràcia, Marta, Maria-Josep Jarque, Marta Astals y Kholoud Rouaz (2020), "Desarrollo y evaluación de la competencia comunicativa en la formación inicial de maestros", Revista Iberoamericana de Educación Superior (RIES), vol. XI, núm. 30, pp. 115-136, https://doi. org/10.22201/iisue.20072872e.2020.30.591 [Consulta: fecha de última consulta]. 